\title{
Core y sistema de control neuro-motor: mecanismos básicos para la estabilidad del raquis lumbar
}

CDD. 20.ed. 152.3

796.023

http://dx.doi.org/10.1590/S1807-55092014005000005

\author{
Víctor SEGARRA* \\ Juan Ramón HEREDIA ${ }^{* * * * * * *}$ \\ Guillermo PEÑA* \\ Matías SAMPIETRO*/**** \\ Mauricio MOYANO*/**** \\ Fernando $\mathrm{MATA}^{* * * *}$ \\ Felipe ISIDRO ${ }^{* * * * *}$ \\ Fernando MARTÍN* \\ Marzo Edir DA SILVA-GRIGOLETTO ${ }^{* * *}$
}

*Instituto Internacional Ciencias Ejercicio Físico y Salud - Espanha. ${ }^{* *}$ Asociación Scientific Sport - Espanha.

***Physical Exercise \& Health Consulting - Espanha.

${ }^{* * * * E q u i p o ~ P h y s i c a l ~-~}$ Argentina.

*****Universidad Valencia - Espanha

******Centro de Ciências Biológicas e da Saúde, Universidade Federal de Sergipe.

\section{Resumen}

El "CORE" es un concepto funcional que engloba la integración de tres sistemas cuyo óptimo funcionamiento garantiza la realización de tareas con una mayor eficacia y seguridad a nivel raquídeo, permitiendo adecuados niveles de estabilidad y control del movimiento. En este sentido, a fin de afrontar con éxito retos que demanden un control dinámico de la columna y la pelvis, el SNC debe aplicar estrategias diferentes, sopesando as fuerzas internas y externas con el fin de proporcionar una respuesta muscular que permita un movimiento óptimo y resista cualquier posible perturbación. En el presente manuscrito se revisa de forma aplicada, las bases, atendiendo a la información disponible actualmente, de los mecanismos básicos de control motor y las posibles alteraciones en los mismos a ser considerados por los especialistas en ejercicio respecto a su intervención mediante programas de ejercicio para la mejora de la capacidad de estabilización raquídea.

Palabras-Clave: Raquis; Estabilidad; Feedforward; Dolor lumbar.

\section{Introducción}

Se asume que el término CORE fue usado por primera vez por Richard H. Dominguez, Robert S. Gajda en su libro Total Body Training ${ }^{1}$. Etimológicamente CORE significa núcleo, centro o zona media. Desde el punto de vista de la actividad física el "CORE" hace referencia al complejo muscular situado en la parte central del cuerpo (región lumbo-pélvica) que incluye 29 músculos que estabilizan la columna vertebral y la región abdominal e incluye músculos del abdomen, espalda, parte posterior y anterior de la cadera, suelo pélvico y diafragma ${ }^{2-4}$. La acción conjunta de estas estructuras permite un adecuado control de la estabilidad corporal ${ }^{5-6} \mathrm{y}$ de la ejecución de tareas que realizan los miembros superiores e inferiores, de forma combinada o secuencial ${ }^{5,7-8}$. Por lo tanto, más que un concepto puramente anatómico, debe ser entendido como un concepto funcional que engloba estructuras musculares, osteo-ligamentosas y de control neural relacionadas con la región mencionada?.

En consecuencia, el entrenamiento del CORE constituye no solo un elemento central y clave para el desempeño de la mayoría de las actividades de la vida diaria (AVD), vida laboral (AVDL) y deportivas (AVDe), sino que también será condición necesaria para progresar en la utilización de ejercicios multiarticulares o aquellos que son ejecutados con elevadas resistencias. Para todas estas tareas el CORE es el centro de la cadena cinética funcional ${ }^{10}$.

En la literatura existen amplios y destacables aportes en torno al entrenamiento del CORE, tanto desde una perspectiva rehabilitadora como desde el rendimiento deportivo, siendo mucha de esa información 
extrapolada y utilizada, con mayor o menor acierto, en el diseño de programas de entrenamiento. Sin embargo los criterios específicos que deben ser tenidos en cuenta para generar un adecuado acondicionamiento y potenciación del CORE parecen ser poco claros, en especial cuando se quiere hacer referencia a las progresiones destinadas a lograr adaptaciones que permitan garanticen la optimicen la estabilización del raquis. Esta situación hace recomendable establecer criterios claros sobre el entrenamiento de esta zona del cuerpo, y proponer una adecuada progresión integrada en los programas de acondicionamiento físico en personas sanas) para poder progresar en las demandas de la AVD, AVDL y AVDe.

Desde esta perspectiva, debemos concebir el entrenamiento lumbo-abdominal (CORE), como base fundamental de los programas de entrenamiento saludable y su desarrollo será necesario para realizar tareas de manera eficaz y con el riesgo de lesión menos acentuado ${ }^{11}$. Esto supone garantizar un óptimo estado y funcional del sistema pasivo, del sistema muscular y del sistema de control motor con la finalidad de satisfacer los requisitos del equilibrio postural (estático y dinámico), crear movimientos específicos, soportar fuerzas externas inesperadas o generar presión con la que ayudar a la respiración dificultosa ${ }^{11}$. Para satisfacer los retos de un control adecuado de la columna y la pelvis, el SNC debe aplicar estrategias diferentes con las que regular el movimiento del tronco. Para ello, el SNC debe ajustar cuidadosamente las fuerzas internas y externas con un patrón muscular que facilite el movimiento en la trayectoria deseada y, al mismo tiempo, resistir cualquier perturbación ${ }^{12}$.

Estas estrategias pueden estar asociadas con el riesgo, real o percibido, que pueda sufrir la columna vertebral ${ }^{11}$. Los elementos que pueden influir en la selección de la estrategia podrían comprender, entre otros, los siguientes aspectos: a) fuerzas que actúan sobre la columna vertebral; b) previsibilidad del movimiento que se va a ejecutar; c) disponibilidad de información (¿capacidad?) propioceptiva adecuada; d) posibilidad real o percibida de lesión y/o dolor; e) experiencia previa disponible ${ }^{11}$.

Por todo ello, el objetivo de este trabajo es exponer los aspectos más relevantes respecto a: 1) mecanismos básicos relacionados con el control neural de la estabilidad del tronco; 2) diferencias del control motor del CORE en sujetos de diferente condición física (deportistas vs. no deportistas); 3) estrategias de control motor del CORE en sujetos sanos y su relación con el dolor lumbar crónico y otras patologías asociadas; 4) ofrecer directrices útiles para su implementación en programas de acondicionamiento físico saludable.

\section{Mecanismos básicos y posibles alteraciones relacionadas con el sistema de control de la estabilidad del tronco}

Toda tarea motriz implica la necesidad de activar mecanismos de control y regulación del movimiento, que son de naturaleza nerviosa, y que serán más complejos cuanto mayor sea la dificultad de la tarea a realizar. El control del sistema neuromuscular depende directamente del sistema sensoriomotor y puede ser entendido como la facultad que tiene un sujeto para efectuar movimientos complejos de forma eficaz y con un mínimo de energía. Este complejo sistema incorpora todos los receptores y vías aferentes (percepción), así como los mecanismos de integración y procesamiento central (decodificación, planificación, y programación) y las respuestas eferentes necesarias (ejecución), para poder mantener la estabilidad funcional durante tareas que se desean realizar ${ }^{13}$.

En función de la complejidad del gesto, el control y la regulación de un movimiento, afectará diferentes estructuras e interacciones del sistema nervioso, las cuales varían en función de cual sea sus características y grado de dificultad. Esto es algo que tiene lugar durante el trabajo muscular más elemental (correr, pedalear, etc.), cuando se quieren ejecutar estructuras técnicas cerradas y automatizadas o incluso durante la realización de gestos deportivos de elevada complejidad.

En cada caso, el SNC debe determinar las estrategias idóneas para satisfacer un patrón motor adecuado que garantice una adecuada estabilidad de la columna, incluso de forma anticipada, para poder emprender una respuesta rápida proporcional a la perturbación generada. Tales situaciones se basan en un complejo mecanismo de biofeedback donde la capacidad propioceptiva es determinante para establecer la posición exacta y el movimiento de la columna lumbar y pelvis, mediante modelos internos que garantizan la interacción entre el cuerpo y las fuerzas operativas ${ }^{11}$. Desde el punto de vista anatómico y funcional, los nervios vinculados 
a los movimientos permiten la intervención de un complejo número de estructuras que quedan englobadas en lo que se conoce como sistema de control y dirección de los movimientos que están unidos entre sí y con el medio circundante por una compleja red de canales de enlace de doble sentido (directo e inverso) entre las diferentes estructuras del sistema nervioso central y periférico. De forma secuencializada y especializada, se llevan a cabo las funciones de percepción, decodificación, planificación, programación y ejecución de los movimientos. Asi como su corrección permanente.

Estos mecanismos suelen iniciarse en los receptores sensoriales ${ }^{14}$ (exteroceptores, propioceptores, interoceptores, y teleceptores), que, vía aferente, transmiten información al córtex y otras zonas del encéfalo a través de las vías aferentes, se identifican, asocian y programan en las estructuras del sistema nervioso central y son enviados a la musculatura más adecuada a través de las vías eferentes.

La información aferente pueda ser redundante a través de tres fuentes sensoriales principales: somatosensorial, visual y vestibular. Todas ellas deben actuar en perfecta coordinación, los receptores musculares del sistema somatosensorial constituyen la estructura que más se puede modificar con el entrenamiento deportivo ${ }^{15}$.

La información aferente de la que hablamos puede ser procesada e integrada en tres niveles de control motor: médula espinal (respuestas muy rápidas y/o reflejas), tronco cerebral (respuestas intermedias y/o automáticas) y corteza cerebral (más lentas, más elaboradas y son voluntarias $)^{11}$.

Podemos definir feedback (retroalimentación) como la elaboración de una respuesta correctiva dentro de un gesto motor como consecuencia de haber percibido anomalías o cambios repentinos del entorno. Dicho de otro modo, se refiere a la información proporcionada, bien por vía refleja o no, después del análisis de un determinado estímulo sensorial ${ }^{15}$.

El feedforward se define como las acciones anticipatorias/preventivas que ocurren antes de la detección sensorial de una disrupción de la homeostasis con base en experiencias anteriores ${ }^{15}$. Los ajustes de anticipación postural subyacen a la capacidad del sistema nervioso de contrarrestar las fuerzas de reacción inducidas por un movimiento focal antes de efectuar el propio movimiento ${ }^{16}$. En este contexto, el músculo transverso abdominal ( $\operatorname{Tr} \mathrm{A})$ es uno de los componentes de la musculatura profunda abdominal que están involucrados en el control del tronco ${ }^{17}$, contribuyendo en los ajustes posturales anticipatorios del control segmentario de la columna lumbosacra durante movimientos focales $^{18}$. En ese sentido, se ha comprobado la activación previa del transverso abdominal durante cualquier dirección de movimiento del miembro superior ${ }^{19-20}$ y, parece ser que el resto de músculos abdominales tienen una activación previa solo en algunas direcciones, de acuerdo con su posición anatómica ${ }^{19-20}$ y la dirección de la demanda de estabilización que se pueda generar. Las fibras horizontales del TrA y sus inserciones en la fascia toracolumbar permiten un control fino de la región lumbopélvica por una acción directa sobre las vértebras lumbares ${ }^{21}$ y el aumento de la presión intraabdominal ${ }^{22}$. Por el contrario, los músculos abdominales superficiales ayudan a la rigidez y en el papel dinámico a la hora de generar movimiento para la columna vertebral.

\section{Control motor del core en deportistas y no deportistas}

Es un hecho que la estabilidad del complejo lumbo-pélvico resulta un factor importante en la realización eficiente de habilidades motoras deportivas ${ }^{4}$. En consecuencia, parecería sería lógico pensar que los deportistas presenten estrategias de activaciones neuromotoras diferentes, o más desarrolladas, que los no deportistas, e incluso que estas estrategias estén en relación a la especificidad de la demanda de estabilización que imponen estos deportes sobre el raquis.

Todo parece indicar que la aplicación de cargas de inestabilidad súbita que actúen sobre el tronco de manera imprevista, podrían generar adaptaciones que mejoren el tiempo de respuesta y el control del desplazamiento del mismo ${ }^{19-20}$. Poblaciones de mujeres no entrenadas que eran expuestas a desestabilizaciones súbitas del tronco durante la realización de juegos reducidos de fútbol, mejoraban el tiempo de respuesta de los músculos del CORE y el control del desplazamiento (ante desestabilizaciones súbitas del tronco) en comparación a un grupo de mujeres que realizaban carrera continua y con respecto a un grupo control ${ }^{23}$. Por lo tanto, la especificidad del deporte podrían resultar un factor determinante a la hora de generar adaptaciones 
positivas en las estrategias de control motor del tronco que pueden ser transferidas a las demandas de estabilización que el deporte genera sobre el mismo.

En un interesante estudio ${ }^{24}$ realizado con 277 deportistas (140 mujeres y 137 hombres) se valoró, de forma longitudinal (3 años), la capacidad de estabilización raquídea en diferentes planos ante una situación inesperada. En el estudio, se pudo comprobar como un déficit en el control neuromuscular del raquis se relacionaba con un mayor riesgo de lesión en la articulación de la rodilla, especialmente en la muestra de mujeres.

En esta misma línea, podemos observar como existen diferencias en la estrategia de activación de los músculos estabilizadores del CORE entre jugadores de fútbol y no jugadores ${ }^{25}$. En este trabajo, los jugadores de fútbol presentaron menores tiempos de latencia motora ante desestabilizaciones súbitas del tronco en el plano sagital. Una posible explicación podría ser que aquellos deportes con fuertes desaceleraciones y cambios de dirección y/o sentido generan cargas desestabilizantes del tronco que deben ser compensadas por los músculos estabilizadores del núcleo obligando al SNC a generar estrategias de estabilización que son específicas para dichas cargas.

En consecuencia, nos parece importante considerar estas adaptaciones diferenciadas en los procesos de estabilización del CORE cuando se desean planificar progresiones de ejercicios entre las diferentes poblaciones de deportistas. Por lo tanto, parece claro que no es recomendable proponer trabajos de fortalecimiento del CORE sin considerar las demandas específicas de cada actividad deportiva ${ }^{26}$.

\section{Control motor del ore en sujetos sanos y su relación con el dolor lumbar crónico y otras patologías}

En sujetos sanos, la activación previa del $\operatorname{Tr} A$, feedforward, parece producirse antes de la realización de tareas funcionales (movimiento del brazo o pierna) ${ }^{18-19}$. Estos mecanismos parecen ser independientes de la velocidad, peso y dirección del movimiento ${ }^{20}$ y de la activación de otros músculos abdominales superficiales ${ }^{27-28}$. No obstante, cuando se realiza una flexión explosiva del hombro en posición erecta, se ha visto una activación previa del transverso contralateral al brazo ejecutor respecto al transverso ipsolateral, lo que nos indica que ambas partes tienen un rol diferente en los ajustes de anticipación postural en determinadas acciones motrices ${ }^{29}$. Este hallazgo podría llevar a pensar que la asimetría de activación del transverso abdominal, durante ajustes posturales anticipatorios, refuta el concepto de corset abdominal anticipatorio (al producirse esta diferencia en la activación anticipadora), no obstante en este caso cabría pensar que dicha anticipación previa del TrA contralateral podría cumplir el rol de controlar el torque de rotación del tronco ${ }^{29-30}$. Por otra parte, en sujetos que reportan dolor lumbar la activación del TrA se ve retardada ${ }^{27}$. En consecuencia, se observa de forma clara en el músculo $\operatorname{Tr} A$ contralateral ${ }^{18,31}$ o transverso ipsilateral y oblicuo interno durante movimiento rápido ${ }^{32}$.

Por otro lado se ha observado en sujetos sanos (con feedforward del TVA inalterado en la flexión de hombro) que al inducirles dolor por infiltración de suero hipertónico, se traducía un retraso en la activación del TrA posterior a la aparición del dolor $^{33}$. Por lo que no está claro si el dolor lumbar retrasa la activación del TVA o el retraso en la activación del transverso es el causante del dolor lumbar ${ }^{34}$. Sin embargo, sí se han observado cambios en el reclutamiento muscular en personas con dolor lumbar inducido por la infiltración de suero hipertónico cuando estos sujetos son evaluados con Estimulación Magnética Transcraneal (EMT) ${ }^{35}$. En pacientes con recidiva de dolor lumbar, se detectaron, con una sola sesión de entrenamiento (3 series de 10 repeticiones de 10 segundos) del transverso abdominal, cambios instantáneos en el timming de reclutamiento de éste músculo, lo que nos indica una mejora del feedforward del $\operatorname{Tr} \mathrm{A}^{36}$.

Desde un enfoque neurofisiológico, el retraso en el transverso contralateral en el ajuste postural anticipatorio va asociado a alteraciones en la plasticidad de la corteza motora primaria $(\mathrm{M} 1)^{37-38}$. Es decir, un cambio en la representación de M1 TrA se relaciona con el retraso en su activación en pacientes con dolor lumbar ${ }^{37}$.

Se podría concluir que el TrA y el oblicuo interno parecen contribuir a controlar la zona lumbar de manera bilateral durante los movimientos. Sin embargo, en pacientes con dolor lumbar crónico, se detectan cambios significativos en los patrones de ajuste postural anticipatorio tanto en el TrA como en el oblicuo interno que pueden ser el resultado de una disfunción de los mecanismos que subyacen la 
corteza motora primaria en su vinculación con el control de la estabilidad de la columna ${ }^{16}$.

La musculatura respiratoria también es reclutada por el SNC para satisfacer las demandas de estabilidad. Así la actividad del diafragma y del TrA se inicia antes de los movimientos rápidos del miembro superior $^{20}$ y se mantiene tónicamente activada durante los movimientos iterativos del miembro superior ${ }^{39} \mathrm{y}$ la march $a^{40}$. También, la actividad de la musculatura del suelo pélvico parece contribuir a los mecanismos de control postural en los movimientos asociados a perturbaciones de la columna que son generados por gestos que involucran al miembro superior ${ }^{41}$.

En torno a esta cuestión, algunas técnicas que han emergido con fuerza en los últimos años, como las denominadas "hipopresivas" desarrolladas por el Dr. Marcel Caufriez, utilizan argumentos en torno a una hipotética relajación del diafragma, activación del TrA y musculatura del suelo pélvico ${ }^{42}$, basada en diferentes acciones que suponen la realización de una "apnea espiratoria" en combinación con determinadas posturas ${ }^{43}$.

$\mathrm{Si}$ bien es cierto que en algunos estudios parece mostrarse una elevación significativa del nivel de activación del $\operatorname{Tr} A$ mediante estos procedimientos metodológicos ${ }^{44}$, se debe considerar que algunos especialistas de estas técnicas critican este estudio por la metodología utilizada ${ }^{45}$. Al parecer, en la mencionada investigación no se utilizó el método en base a unos criterios técnicos adecuados ${ }^{42}$. A este respecto, es difícil justificar como válido la mayor activación de cierta musculatura clave en la estabilización del raquis lumbar y a continuación cuestionar la metodología empleada en la intervención con uno de los grupos, cuando las conclusiones no muestran mejoras significativas al utilizar tales métodos.

Dado que, según estos especialistas, no fue utilizada la técnica original, creemos que quizás utilizaron un "abdominal hollowing", lo que podría explicar dicho resultado (mayor activación del TrA). No obstante, no seremos nosotros quienes pongamos en duda, ni cuestionemos, los trabajos publicados por autores del nivel de Stupp y su grupo de colaboradores ${ }^{44}$.

También en las bases teóricas y la fundamentación de las técnicas hipopresivas, se argumenta la existencia de una hipotética respuesta "refleja" (es decir involuntaria y mediada por el sistema nervioso) relacionada con la estimulación "propioceptiva" vinculada a las posturas adoptadas y la apnea respiratoria que provoca un estado cercano a la hipercapnia (estimulación centros respiratorios del tronco cerebral - centro pneumotáxio y centro respiratorio bulbar ventral - e inhibición de los inspiratorios - centro apnéustico y centro respiratorio bulbar dorsal). En esta situación se genera una disminución en la actividad tónica (con la consiguiente relajación) del diafragma con disminución de la presión intraabdominal que provocaría, vía refleja, una "tonificación" de la faja abdominal y la musculatura perineal, a la vez que provocaría una succión de las vísceras situadas en la región pélvica con disminución de la tensión ligamentosa ${ }^{46}$. Caso de que esto fuese así, sería de enorme interés en determinados programas de entrenamiento y con algunos sujetos específicos, convirtiéndose en una valiosa herramienta para lograr objetivos relacionados con el control neuro-motor del CORE (que es el tema abordado en el presente artículo).

Respecto a esta cuestión, y tras un análisis de la literatura existente, no encontramos estudio alguno que corrobore dicha hipótesis. Debe ser tenido en cuenta que, a nivel fisiológico, el argumento es discutible y la bibliografía utilizada como apoyo a tales afirmaciones ${ }^{47-48}$ no proporcionan un sustento sólido para ser mantenidas.

En consecuencia, encontramos algunas dificultades para poder otorgar a estas afirmaciones el rigor científico necesario para asegurar que ésta metodología pueda ser utilizad con garantía en el entrenamiento de la capacidad de control y estabilización raquídea. Entendemos necesario una revisión de los principios básicos y fundamentación, y ser cautelosos respecto a los potenciales beneficios de la propuesta. Es necesario esperar a que las investigaciones que puedan ser desarrolladas en torno a estas propuestas puedan aportar más luz y evidencias respecto a esta cuestión, y brindar, de esta manera, una herramienta más al servicio de los programas de acondicionamiento físico saludable.

Llegados a este punto, resulta interesante considerar que los modelos estáticos de regulación de la columna no tienen en cuenta la interacción entre el control de la misma, la ventilación y la continencia. En estos modelos, no es habitual que se consideren las estrategias que emplea el SNC para regular la estabilidad espinal y, por lo tanto, habría que contemplar que los déficits de la respiración y de la continencia pueden mermar el control de la columna vertebral ${ }^{12}$. Este aspecto deberá ser considerado, tanto a la hora de valorar posibles disfunciones, como para establecer progresiones adecuadas y estrategias metodológicas que contemplen y reduzcan la posibilidad de provocar conflictos funcionales que son claves para la salud y la integridad raquídea. 


\section{Recomendaciones finales}

Tras esta revisión e interpretación de la información actual existente, podemos detallar algunas recomendaciones de utilidad a la hora implementar programas de acondicionamiento físico saludable:

- La actividad tónica del TrA ha sido observada en sujetos sanos durante la marcha ${ }^{49}$, algo que no ocurre en sujetos con dolor lumbar donde se produce una activación fásica ${ }^{50}$. Como estos cambios persisten tras la remisión de los síntomas ${ }^{18}$ los déficits en el control de la musculatura abdominal han sido asociadas con la persistencia y cronicidad del dolor lumbar ${ }^{33}$;

- Se ha observado que el entrenamiento para mejorar el control de la musculatura del tronco es efectivo para mejorar la activación muscular durante tareas funcionales ${ }^{51-52}$. Cambios en los mecanismos de control neural de la musculatura del tronco también contribuye al dolor lumbar ${ }^{53}$;

- Parece existir un retraso en la activación del TrA durante movimientos del brazo ${ }^{18} \mathrm{y}$ durante la marcha ${ }^{50}$, lo que supone un marcador de disfunción de los músculos del tronco ${ }^{34}$. En consecuencia, este comportamiento deberá ser considerado al diseñar los programas de acondicionamiento físico. A este respecto, todo parece indicar que la actividad del músculo $\operatorname{Tr} \mathrm{A}^{18-19}$ y los multífidos $^{54}$ se retrasa durante la realización de movimientos rápidos que involucra a la articulación del hombro y de las piernas en personas que padecen dolor lumbar;

- Estudios controles aleatorizados, muestran que los programas bien estructurados de entrenamiento cuyo objetivo es la mejorara el control de la musculatura del tronco, conlleva una mejora significativa de la sintomatología relacionada con el dolor lumbar ${ }^{5-57}$;

- Atendiendo a esta información, todo parece aconsejar la realización de un adecuado "screening" y valoración de los sujetos, para garantizar progresiones adecuadas de integración neuromuscular de la musculatura del CORE. Estas intervenciones deben prestar especial atención, en las primeras fases (mediante entrenamientos específicos), al uso de estímulos y maniobras que enfaticen en un adecuado nivel de activación de la musculatura profunda del CORE (especialmente TrA, OI). Tras esta primera fase, parece aconsejable emplear estímulos más globales que garanticen adecuados niveles de co-activación general de las citadas estructuras musculares;

- No hay un único ejercicio que estimule global y funcionalmente toda la musculatura del CORE, por lo que parece clave el profundizar en factores relacionados con la prescripción de los movimientos y las dosis de entrenamiento adecuada para lograrlo.

Por ultimo, parece adecuado atender a las evidencias que irán emergiendo en el futuro, fruto de la intensa investigación que en la actualidad se está realizando sobre la temática analizada. La información resultante nos permitirá extraer información relevante que permita optimizar los criterios adecuados para el desarrollo de programas de entrenamiento dirigidos a la mejora de la capacidad de estabilización raquídea en sujetos sanos, deportistas y no deportistas, y en sujetos con patología lumbar.

\begin{abstract}
Core and neuromotor control system: basic mechanisms for the stability of the lumbar spine

The "CORE" is a funcional concept that englobes the integration of three systems which optimal operation guarantees better eficiency and security in tasks related with the spine, allowing appropiate stability and movement control levels. In order to successfully addres challenges which demand a dynamic control of the spine and the pelvis, the SNC must use diferent strategies, weighing the internal and external forces in order to provide a muscular response to allow an appropiate movement and resist any possible disturbance. This article reviews the foundations based on the information currently available about the basic mechanisms of motor control and posible changes in them, to be considered by exercise specialists regarding to their exercise intervention programs to improve spinal stabilization capacity.
\end{abstract}

KEY woRDS: Raquis; Stability; Feedforward; Back pain. 


\section{Referencias}

1. Dominguez R, Gajda R. Total body training. New York: Warner; 1983.

2. Willardson JM. Core stability training: applications to sports conditioning programs. J Strength Cond Res. 2007;21:979-85.

3. Faries M, Greenwood M. Core training: stabilizing the confusion. Strength Cond J. 2007;29:10-25.

4. Hibbs A, Thompson K, French D, et al. Optimizing performance by improving core stability and core strength. Sports Med. 2008;38:995-1008.

5. Kibler WB, Press J, Sciascia A. The role of core stability in athletic function. Sports Med. 2006;36:189-98.

6. Borghuis J, Hof AL, Lemmink KA. The importance of sensory-motor control in providing core estability: implications for measurament and training. Sports Med. 2008;38:893-916.

7. Stephenson J, Swank AM. Core training: designing a program for anyone. Strength Cond J. 2004; 26:34-7.

8. Tse M, McManus A.M, Masters R.S. Development and validation of a core endurance intervention program: implications for performance in college-age rowers. J Strength Cond Res. 2005;19:547-52.

9. Panjabi MM. The stabilizing system of the spine. Part II: neutral zone and instability hypothesis. J Spinal Disord. 1992;5:390-7.

10. Akuthota V, Nadler SF. Core strengthening. Arch Phys Med Rehabil. 2004;85(Suppl 1):S86-92.

11. Vleeming A, Mooney V, Stoeckart R. Movement stability and lumbopelvic pain: integration of research and therapy. London: Churchill Livingstone; 2007.

12. Hodges PW, Cholewicki J. Functional control of the spine. In: Vleeming A, Mooney V, Stoeckart R. Movement, stability and lumbopelvic pain. London: Churchill Livingstone; 2007. p.489-512.

13. Fort Vanmeerhaeghe A, Romero Rodriguez D. Análisis de los factores de riesgo neuromusculares de las lesiones deportivas. Apunts Med Esport. 2013. Disponible en: http://dx.doi.org/10.1016/j.apunts.2013.05.003.

14. Leonard CT. The neuroscience of human movement. St Louis: Mosby-Year Book; 1998.

15. Riemann BL, Lephart SM. The sensorimotor system. Part I: the physiologic basis of functional joint stability. J Athl Train. 2002;37:71-84.

16. Massé-Alaire H, Flamand VH, Moffet H, Schneider C. Corticomotor control of deep abdominal muscles in chronic low back pain and anticipatory postural adjustments. Exp Brain Res. 2012;218:99-109.

17. Tesh KM, Dunn JS, Evans JH. The abdominal muscles and vertebral stability. Spine (Phila Pa 1976). 1987; 12:501-8

18. Hodges PW, Richardson CA. Inefficient muscular stabilisation of the lumbar spine associated with low back pain: a motor control evaluation of transversus abdominis. Spine. 1996;21:2640-50.

19. Hodges PW, Richardson CA. Contraction of the abdominal muscles associated with movement of the lower limb. Phys Ther. 1997; 77:132-44.

20. Hodges PW, Richardson CA. Feedforward contraction of transversus abdominis is not influenced by the direction of arm movement. Exp Brain Res. 1997;114:362-70.

21. Urquhart DM, Barker PJ, Hodges PW, Story IH, Briggs CA Regional morphology of the transversus abdominis and obliquus internus and externus abdominis muscles. Clin Biomech (Bristol, Avon). 2005;20:233-41.

22. Cresswell AG, Grundstrom H, Thorstensson A. Observations on intraabdominal pressure and patterns of abdominal intra-muscular activity in man. Acta Physiol Scand. 1992;144:409-18.

23. Pedersen, MT, Randers, MB, Skotte, JH, Krustrup, P. Recreational soccer can improve the reflex response to sudden trunk loading among untrained women. J Strength Cond Res. 2009;23:2621-6.

24. Zazulak BT, Ponce PL, Straub SJ, Medvecky MJ, Avedisian L, Hewett TE. Gender comparison of hip muscle activity during single-leg landing. J Orthop Sports Phys Ther. 2005;35:292-9.

25. Borghuis AJ, Lemmink, Hof A. Core muscle response times and postural reactions in soccer players and nonplayers. Med Sci Sports Exerc. 2011;43:108-14.

26. Shinkle J, Nesser TW, Demchak TJ, McMannus DM Effect of core strength on the measure of power in the extremities. J Strength Cond Res. 2012;26:373-80.

27. Hodges PW. Changes in motor planning of feedforward postural responses of the trunk muscles in low back pain. Exp Brain Res. 2001;141:261-6.

28. Hodges PW, Richardson CA. Transversus abdominis and the superficial abdominal muscles are controlled independently in a postural task. Neurosci Lett. 1999;265:91-4.

29. Allison GT, Morris SL Transversus abdominis and core stability: has the pendulum swung? Br J Sports Med 2008; 42:930-1.

30. Radebold A, Cholewicki J, Panjabi MM, Patel TC. Muscle response pattern to sudden trunk loading in healthy individuals and in patients with chronic low back pain. Spine. 2000;25:947-54.

Rev Bras Educ Fís Esporte, (São Paulo) 2014 Jul-Set; 28(3):521-29 • 527 
31. Hodges PW, Richardson CA. Delayed postural contraction of transversus abdominis in low back pain associated with movement of the lower limb. J Spinal Disord. 1998;11:46-56.

32. Silfies SP, Mehta R, Smith SS, Karduna AR. Differences in feedforward trunk muscle activity in subgroups of patients with mechanical low back pain. Arch Phys Med Rehabil. 2009;90:1159-69.

33. Hodges PW, Moseley GL, Gabrielsson A, Gandevia SC. Experimental muscle pain changes feedforward postural responses of the trunk muscles. Exp Brain Res. 2003;151:262-71.

34. Radebold A, Cholewicki J, Panjabi MM, Patel TC. Muscle response pattern to sudden trunk loading in healthy individuals and in patients with chronic low back pain. Spine. 2000;25(8):947-54.35.

36. Tsao H, Hodges PW. Immediate changes in feedforward postural adjustments following voluntary motor training. Exp Brain Res. 2007;181:537-46.

37. Tsao H, Galea MP, Hodges PW Reorganization of the motor cortex is associated with postural control deficits in recurrent low back pain. Brain. 2008;131:2161-71.

38. Jacobs JV, Henry SM, Nagle KJ Low back pain associates with altered activity of the cerebral cortex prior to arm movements that require postural adjustment. Clin Neurophysiol. 2010;121:431-40.

39. Hodges PW, Gandevia SC Changes in intraabdominal pressure during postural and respiratory activation of the human diaphragm. J Appl Physiol. 2000;89:967-76.

40. Saunders SW, Coppieters M, Hodges PW. Reduced tonic activity of the deep trunk muscle during locomotion in people with low back pain. 5th Interdisciplinary World Congress of Low Back and Pelvic Pain; 10-13 nov. 2004; Melbourne, AU. Melbourne: Andry Vleeming; 2004.

41. Hodges PW, Sapsford R, Pengel LHM. Postural and respiratory functions of the pelvic floor muscles. Neurourol. Urodynam. 2007;26:362-71.

42. Rial T, Pinsach P. Principios técnicos de los ejercicios hipopresivos del Dr. Caufriez. [citado 20 dec 2013]. Lec Educ Fis Deportes. 2012;172. Disponible en: http://www.efdeportes.com/efd172/los-ejercicios-hipopresivos-del-dr-caufriez.htm.

43. Rial T, Riera T. Prevalencia y abordaje desde el ejercicio físico de la incontinencia urinaria en mujeres deportistas. [citado 20 dec 2013]. Lec Educ Fis Deportes. 2012;164. Disponible en: http://www.efdeportes.com/efd164/incontinenciaurinaria-en-mujeres-deportistas.htm.

44. Stüpp, L. y Cols. Pelvic floor muscle and transversus abdominis activation in abdominal hypopressive technique through surface electromyography. Neurourol Urodyn. 2011;30:1518-21.

45. Rial T, Álvaez M: Mitos y realidades en torno al método hipopresivo Caufriez. [citado 20 oct 2013]. Lec Educ Fis Deportes. 2012;175. Disponible en: http://www.efdeportes.com/efd175/en-torno-al-metodo-hipopresivo-caufriez.htm.

46. Rial T, Villanueva C, Fernández I. Aproximación conceptual y metodológica al método hipopresivo. [citado 20 oct 2013]. Lec Educ Fis Deportes. 2011;162. Disponible en: http://www.efdeportes.com/efd162/aproximacion-al-metodohipopresivo.htm.

47. Hodges P, Heijnen I, Gandevia S. Postural activity of the diaphragm is reduced in humans when respiratory demand increases, J Physiol. 2001;537:999-1008.

48. Hodges M, Forster H, Papanek P, Dwinell M, Hogan G. Ventilatory phenotypes among four strains of adult rats. J Appl Physiol. 2002;93:974-83.

49. Saunders SW, Rath D, Hodges PW. Postural and respiratory activation of the trunk muscles varies with mode and speed of locomotion. Gait Posture. 2004;20:280-90.

50. Saunders SW, Coppieters MW, Hodges PW. Changes in coordination of human locomotor and respiratory movement in low back pain [abstract]. Musculoskeletal Physiotherapy Australia 14th Biennial Conference; 24-16 nov. 2005; Brisbane, AU. Brisbane: Australian Physiotherapy Association; 2005. S10. Available from: http://www.physiotherapy. asn.au/DocumentsFolder/JoP/Collections/eSupplements/2005\%20MPA\%2014th\%20Biennial\%20Conference.pdf.

51. Tsao H, Hodges PW. Immediate changes in feedforward postural adjustments following voluntary motor training. Exp Brain Res. 2007;181:537-46.

52. Tsao H, Hodges PW. Persistence of improvements in postural strategies following motor control training in people with recurrent low back pain. J Electromyogr Kinesiol. 2008;18:559-67.

53. Cholewicki J, Greene HS, Polzhofer GK, Galloway MT, Shah RA, Radebold A. Neuromuscular function in athletes following recovery from a recent acute low back injury. J Orthop Sports Phys Ther. 2002;32:568-75.

54. MacDonald D, Moseley GL, Hodges PW 2004 The function of the lumbar multifidus in unilateral low back pain. 5th Interdisciplinary World Congress of Low Back and Pelvic Pain; 10-13 nov. 2004; Melbourne, AU. Melbourne: Andry Vleeming; 2004. 
55. Hayden JA, van Tulder WM, Tomlinson G. Systematic review: strategies for using exercise therapy to improve outcomes in chronic low back pain. Ann Intern Med. 2005;142:765-75.

56. Goldby LJ, Moore AP, Doust J, Marion T. A randomised controlled trial investigating the efficiency of musculoskeletal physiotherapy on chronic low back pain disorder. Spine. 2006;31:1083-93.

57. Ferreira PH, Ferreira ML, Maher CG, Herbert RD, Refshauge K. Specific stabilisation exercise for spinal and pelvic pain: a systematic review. Aust J Physiother. 2006;52:79-88.

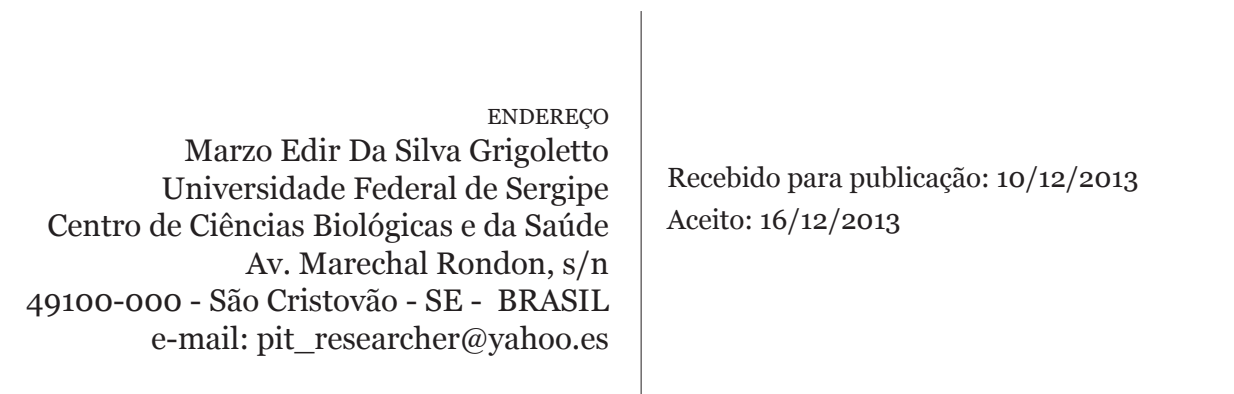

